\title{
The Impact of Background Radio and Television on High School Students' Homework Performance
}

\author{
By Marina M. Pool, Cees M. Koolstra, and Tom H. A. van der Voort
}

This study examined the impact of background media on students' performance and time spent on two types of homework assignments: paper-and-pencil and memorization assignments. Eighth-grade students $(N=160)$ did the assignments with either (a) a soap opera, (b) music videos, (c) radio music, or (d) no medium in the background. Results indicated that doing homework combined with watching a soap opera interfered with students' performance on both types of assignments. However, irrespective of the medium employed, music in the background left homework performance unaffected. There was no indication that background media influenced the amount of time spent to complete bomework assignments.

Survey studies have shown that students regularly combine their homework with listening to the radio or watching television (Beentjes, Koolstra, \& van der Voort, 1996; Patton, Stinard, \& Routh, 1983; Wober, 1992). The most recent survey study demonstrated that about $80 \%$ of the high school students regularly do homework with a radio and 50\% more or less frequently do homework with a television in the background (Beentjes et al., 1996). With so many students combining homework with background media, the question arises whether students' cognitive system is capable of processing the information given in a school assignment as accurately with background media as in a single-task situation without background media.

According to limited-capacity theories, it is likely that the use of background media inhibits homework performance because people have only a limited pool of mental resources for processing information (Basil, 1994; Kahneman, 1973; Lang, 2000). When combining homework and background media, the student is charged with two tasks that compete for the limited information-processing re-

\footnotetext{
Marina M. Pool is a doctoral candidate at the Center for Child and Media Studies at Leiden University, the Netherlands. Cees M. Koolstra (PhD, Leiden University) is an assistant professor at the Center for Child and Media Studies, Leiden University, where Tom H. A. van der Voort (PhD, Leiden University) is professor and head of the Center for Child and Media Studies. The authors wish to thank Linda Franken, Esther Hölscher, Femmy van der Hulst, and Agaath Prins for help in collecting the data. Correspondence should be addressed to Cees M. Koolstra, Center for Child and Media Studies, Leiden University, P.O. Box 9555, 2300 RB Leiden, The Netherlands; e-mail: koolstra@fsw.leidenuniv.nl.
} 
sources. The information presented in the two tasks may exceed the attentional capacity or resources, allowing only part of the information to be processed, and homework performance decreases.

Limited-capacity theorists hold alternative positions when explaining capacity interference between two concurrent tasks (Bourke, Duncan, \& Nimmo-Smith, 1996). One group of theorists argues that interference occurs when the general attentional capacity is exceeded. However, other theorists argue that interference occurs when two tasks compete for the same resources of information processing and the capacity of the specific resources is exceeded, a phenomenon called "structural interference" (Bourke et al., 1996).

When homework is combined with background media, capacity limits may also be exceeded because elements in the structure and content of television can elicit orienting responses (e.g., Lang, 2000). An orienting response involves an automatic allocation of processing resources to the program as a reaction to novel or interesting stimuli, including sound effects, visual complexity, movement, cuts, and zooms (Anderson \& Lorch, 1983; Lang, 2000). The result is that the amount of resources available for the assignments decreases.

Research on the distraction effects of background media mainly employed cognitive tasks. Although findings from these studies were inconsistent, most studies showed that performance on difficult and complex tasks is impaired when the task is executed in combination with background audio stimuli (e.g., Daoussis \& McKelvie, 1986; Furnham \& Bradley, 1997; Graydon \& Eysenck, 1989; Martin, Wogalter, \& Forlano, 1988; Salamé \& Baddeley, 1989) or audiovisual stimuli (e.g., Armstrong, Boiarsky, \& Mares, 1991; Armstrong \& Greenberg, 1990; Furnham, Gunter, \& Peterson, 1994).

According to Pool, van der Voort, Beentjes, and Koolstra (2000), the distraction effects of background media on students' cognitive test performance found in previous studies do not necessarily apply to the home study situation of high school students because (a) the participants were university students instead of high school students, (b) the background television programs used often differed from the programs high school students prefer to watch while doing homework, (c) the cognitive tests were different from homework assignments, and (d) the cognitive tests lasted only a few minutes and had to be done within a limited time, whereas students at home generally have as much time as needed to finish homework tasks.

To our knowledge, only two studies have investigated the impact of background media on actual homework assignments (Cool, Yarbrough, Patton, Runde, \& Keith, 1994; Pool et al., 2000). Results of the Cool et al. (1994) study indicated that neither background radio nor television interfered with homework performance. However, unlike the home study situation, the homework tasks employed in this study had to be done within a limited time. In two Dutch experiments, Pool et al. (2000) found that a background with a Dutch-language soap opera inhibited eighth-grade students' performance on difficult paper-and-pencil assignments without a time limit, whereas English-language music videos did not cause distraction effects. The distraction effect of the soap opera was expressed either in a performance decrement or in an increase in the time used to complete the homework tasks. 
The present study was a continuation of the study by Pool et al. (2000) using the Pool et al. study as the principal framework. The present study had three goals: The first goal was to replicate Pool et al.'s (2000) experiment on distraction effects of background television on difficult paper-and-pencil assignments. As in the previous study, the background programs used were English-language music videos and a Dutch-language soap opera, the two program types Dutch high school students most frequently combine with doing homework (Beentjes et al., 1996). We expected soap operas to cause distraction effects, because both the research conducted with cognitive tasks (Armstrong, 1997; Armstrong et al., 1991; Armstrong \& Greenberg, 1990; Furnham et al., 1994) and with homework assignments (Pool et al., 2000) showed that task performance deteriorated in the presence of soap operas. Consistent with Pool et al.'s findings, we expected that the distraction effects of the soap opera would be expressed either in a decrease in students' performance on difficult paper-and-pencil assignments, an increase in the amount of time used to complete these assignments, or both. Therefore, we hypothesized that:

H1: A soap opera on television will lead to distraction effects on difficult paper-and-pencil assignments.

Pool et al. (2000) observed that background music videos caused neither a performance decrement nor an increase in the amount of time used to complete paper-and-pencil assignments. One reason for this finding may be that the music videos employed in the Pool et al. study used a foreign language, whereas the music used in other studies employed the native language of the participants. The phonological difference between the native language of the participants and the language used in the music videos in the Pool et al. study may have prevented structural interference from occurring. In the present study, again music videos in a foreign language were used, as Dutch high school students are mostly presented with English-language music videos. We anticipated a result similar to the study of Pool et al.:

H2: Foreign-language music videos on television will not lead to distraction effects on difficult paper-and-pencil assignments.

Our second goal was to investigate the impact of background media on memorization assignments because effects on this type of assignment had not been studied before. In addition, we aimed at establishing possible differential effects of background media on paper-and-pencil and memorization tasks. An important difference between the two types of tasks is that memorization assignments require that students be able to answer questions when the study material is no longer available, whereas paper-and-pencil assignments require only the capacity to answer questions while the material is still available. Therefore, the material included in memorization assignments has to be stored into long-term memory and must be easily retrievable. As memorization tasks involve relatively deep levels of processing that can make substantial demands on processing resources 
(Craik \& Tulving, 1975), memorization tasks probably require more processing capacity than paper-and-pencil tasks do. In terms of the limited capacity hypothesis (Kahneman, 1973), capacity limits will therefore be reached sooner when memorization rather than paper-and-pencil tasks are involved. Thus, we expected to find that:

H3: Distraction effects will be stronger for memorization assignments than for paper-and-pencil assignments.

Unlike paper-and-pencil assignments, we expected memorization assignments to suffer from foreign-language music videos because capacity limits will be reached sooner when memorization tasks are involved. However, we expected to find that performance on memorization tasks would suffer less from music videos than from a soap opera (Pool et al., 2000) because soap operas contain more dialogue than music videos do. Soap operas, therefore, are more likely to interfere with students' phonological working memory, which increases the likelihood that structural interference occurs (Salamé \& Baddeley, 1989). In addition, because the soap opera used the students' native language whereas the music videos used a foreign language, structural interference is more likely in the presence of the background soap opera than in the presence of the music videos. We therefore expected to find that:

H4: A soap opera will lead to stronger distraction effects than music videos when memorization assignments are involved.

The third goal of the present study was to compare the impact of background radio and television on homework performance. Differential distraction effects of radio and television on homework performance have been examined in only one study (Cool et al., 1994). However, the study was unable to detect differential effects of background radio and television because the contents of the radio and television programs were not comparable. In the present study, the audio content of the background music television and radio were exactly the same, which enabled us to compare the strength of the distraction effects invoked by the two background media. We expected that background television would lead to a stronger distraction effect than background radio because radio presents only audio information, whereas television presents both audio and visual information. Television therefore has a higher potential for overloading the information-processing capabilities of users (Drew \& Grimes, 1987). In addition, auditory stimuli on television may elicit orienting responses, which direct students' attention to the television screen and, therefore, may increase the time used to complete the assignments. Because we hypothesized that background music television would lead to distraction effects only on memorization assignments, we expected to find differential distraction effects of radio and music television on memorization assignments only. Hence, it was hypothesized that:

H5: Distraction effects on memorization assignments will be stronger for background music videos than for background radio music. 


\section{Method}

\section{Participants}

The study was conducted with a sample of 160 students from Grade 8 (age, $M=$ 14 years 2 months; boys, $n=80$, girls, $n=80$ ) from five secondary schools in the urban district of Leiden, the Netherlands. The five schools included in the study provided general secondary education to prepare students for intermediate or higher vocational school but not for university. The IQs of students who attend this type of secondary school usually lie between 90 and 120. All participants were proficient in Dutch. Participants came from both lower and middle-class families.

Prior to the selection of the participants, a standardized reading comprehension test (van Kan \& Aarnoutse, 1993) was administered to 336 eighth-grade students in their classrooms. At the end of the test, the students were asked to participate in the experiment for a payment of 20 guilders (about 10 dollars) at a future date. Of the 336 students, 260 (77\%) were willing to participate. The decision was made to include 160 participants in the experiment, and to keep the cells even.

\section{Design}

We reduced the 260 students willing to participate to 160 participants in three steps. First, we matched students on gender, resulting in a group of 130 boys and a group of 130 girls. Second, we gave students one point for every correct answer (maximum 30 points) on the classical reading comprehension test (van Kan \& Aarnoutse, 1993) and formed blocks of students with identical reading comprehension scores or scores that were at most two points apart. This resulted in 20 blocks of six or seven girls and 20 blocks of six or seven boys. Finally, from each of the blocks of six or seven students, respectively, we excluded two or three randomly chosen students, resulting in 20 blocks of four girls and 20 blocks of four boys remaining.

The participants included in each block of four students were randomly assigned to one of four conditions in which homework was done with either: (a) a Dutch-language soap opera, (b) English-language music videos, (c) English-language radio music, or (d) no medium in the background. All participants received both paper-and-pencil and memorization assignments. The order of presentation of the paper-and-pencil and the memorization assignments was systematically varied. In addition, the order of the two memorization assignments was systematically varied.

The overall design was a 4 (soap opera vs. music videos vs. radio music vs. silence) X 2 (paper-and-pencil vs. memorization) randomized block design (Kirk, 1982), with background condition as between-subjects factor and type of homework assignment as a within-subjects factor. Error variance could be reduced (Kirk, 1982) because (a) subjects were matched into blocks on the basis of a variable (reading comprehension) that is correlated with the dependent variable $(r=.31 ; p=.000)$; (b) each block had as many subjects as the number of treatment levels; and (c) subjects within each block were randomly assigned to treatment levels, with the result that each of the treatment levels was represented within 
each block. Because data obtained from subjects in the same block could be treated as if they were repeated measures obtained from the same person (Tabachnick \& Fidell, 1996), in the statistical analyses, the factor background condition was treated as a within-subjects factor or, rather, a "within-blocks" factor.

\section{Materials}

Background programs. The videotape used in the soap opera condition contained a highly popular Dutch soap opera called Goede Tijden, Slechte Tijden (Good Times, Bad Times), the same soap opera used in the earlier study (Pool et al., 2000). The videotape comprised eight episodes of the soap opera. Total duration of the videotape was 167 minutes. The episodes were played without interruption by replays of extracts from previous episodes, commercials, or leaders.

The videotape used in the music videos condition consisted of 50 Englishlanguage music videos totaling about 180 minutes. They were taken from popular music programs broadcast about 1 year prior to the experiment. The music videos were selected randomly from programs that presented music videos from the hit parade and included both story/fantasy and concert videos. No rap music or hard rock videos were included. Rapid scene changes and movement characterized the visual content of the music videos. The music videos were not interrupted by interviews, announcements, or television commercials. The audiotape used in the radio music condition contained exactly the same music as the videotape in the music videos condition.

Homework assignments. The paper-and-pencil assignments consisted of two reading comprehension tasks. The first reading comprehension task consisted of five Dutch texts and a total of 25 corresponding multiple-choice questions (Gilissen $\&$ Aarnoutse, 1996). The questions required students to identify the central theme of a text, to link different parts of a text, and to draw inferences from the information provided in the text. The text was available to participants as they answered the questions.

The second reading comprehension task included three English reading texts for which we formulated a total of 19 corresponding English multiple-choice questions. Again, the text was available while the students answered the questions, and the type of questions used was similar to those of the Dutch reading comprehension test. Cronbach's alpha for the composite measure of the paper-and-pencil assignments was .75.

The memorization assignments consisted of both Dutch-language and Englishlanguage tasks. The tasks were originally developed for the present study and addressed topics about which students had little or no prior knowledge.

The Dutch memorization assignment included a geography and a history task. The geography task consisted of two parts. First, the students studied a map with the names of 22 African countries. Recall questions then required students to name 15 African countries shown on an unmarked map. Second, students had to memorize the names of the capitals of eight of these African countries, and recall questions asked them to name the capitals of seven given countries. In the history task, students studied the history of an imaginary African island, then took the recall test that included 16 questions that assessed their factual knowledge about 
the history of the island. The study material was not available when students answered the questions. The two tasks that formed the Dutch memorization assignment required a combination of memory operations. Probably, the geography task tested rote memory whereas the history task was likely to test narrative memory. The geography and history tasks were moderately correlated $(r=.39, p=.000)$.

The English memorization task required students to learn the translation of 17 English words into Dutch and vice versa. Each English-Dutch word pair was followed by a sample sentence. The recall test asked students to translate the Dutch words into English or vice versa. For the memorization assignments combined, Cronbach's alpha was .92.

\section{Procedure}

The experiment was conducted in a university building. Upon arrival at the university, the participant reported to the reception desk and was met by one of five trained experimenters. The experimenter took the participant to one of the four experimental rooms. Rooms were systematically varied across the four conditions. The participants received the experimental treatments individually. The experimenters were randomly assigned to the participants.

Each experimental room was equipped with a writing desk with chair, a small table with comfortable chairs, and a cupboard. The participant was seated at the writing desk, upon which sat a digital clock, a pen, and a beverage for the student. In the television conditions, a television set with a 23-inch screen and a VCR were placed in front of the participant at a distance of approximately 10 feet. In the radio condition, a radio was placed in front of the participant at a distance of approximately 10 feet. The volume of the sound of the radio or television was set at a level of about $60-70$ decibels.

As each student was seated, the experimenter instructed the student about the tasks and asked him or her to write down the time on the clock at the beginning and end of each task. Participants were told that they would be left alone while doing the assignments. This decision was made to increase the similarity between the experimental and home study situation. Subsequently, the participant was asked to notify the experimenter when the tasks in the first booklet were completed, and the participant was shown where the experimenter was to be found.

After the explanation of the first booklet, participants in the two television conditions were given a dual-task instruction because it approximates the way students tend to combine background media with doing homework in the home situation. The participant was told: "A lot of students do their homework in front of the television. Therefore, we'll also turn on the television here. Do the homework just as you do it at home. It is no problem if you want to watch television every so often. I'll turn on the television now." Students in the radio condition received the same instruction.

After the participant had finished the first booklet, the experimenter collected this booklet and explained the tasks in the second booklet. However, if the first booklet involved memorization assignments, students were first presented with the booklet with recall questions. During the recall test, the television or radio was turned off. Students were paid after finishing assignments. 


\begin{tabular}{|c|c|c|c|c|c|}
\hline Homework assignment & Pap & bencil & & tion & \\
\hline \multicolumn{6}{|l|}{ Television } \\
\hline Soap opera & .56 & .13 & .56 & .17 & 40 \\
\hline Music videos & .62 & .14 & .63 & .20 & 40 \\
\hline Radio music & .64 & .13 & .64 & .19 & 40 \\
\hline Silence & .63 & .10 & .67 & .19 & 40 \\
\hline Total & .61 & .13 & .63 & .19 & 160 \\
\hline
\end{tabular}

\section{Results}

Initial data checks showed that the distributions of the two dependent variables (i.e., the proportion of correct answers and the amount of time used to complete homework assignments) met two assumptions underlying analysis of variance, that is, they were normally distributed and the observations were independent. The third assumption, homogeneity of variance, was not met. However, for our data, a violation of this assumption did not have much effect on the error rates because the background conditions had equal sample sizes (Boneau, 1960; FeirWalsh \& Toothaker, 1974; Stevens, 1992). An analysis of outliers using Cook's distances (1977) yielded no data points for which Cook's distance was greater than 1 (Cook \& Weisberg, 1982). Therefore, the scores of all 160 participants were included in the analyses. An alpha level of .05 was used for all statistical tests (unless stated otherwise).

\section{Homework Performance}

Table 1 presents the proportion of correct answers for each background condition and both types of assignments. A 4 (soap opera vs. music videos vs. radio vs. silence) X 2 (paper-and-pencil vs. memorization assignments) analysis of variance with background condition as a "within-blocks" factor yielded no statistically significant main effect for type of homework assignment, $F(1,39)=.80, M S E=.02$, $p=.378, \eta^{2}=.002$, using $\eta^{2}=\mathrm{SS}_{\text {between }} / \mathrm{SS}_{\text {total }}$ (Levine \& Hullett, 2000), indicating that performance scores on the paper-and-pencil and memorization assignments were about equal. As was expected, a significant main effect for background condition emerged, $F(3,117)=4.85, M S E=.03, p=.003, \eta^{2}=.050$. Using a Bonferroni correction resulting in a more stringent level of significance $(p<.008)$, post hoc $t$-tests revealed that overall performance scores of the students in the soap opera condition were significantly lower than those of students in the con- 


\begin{tabular}{|c|c|c|c|c|c|}
\hline \multirow{2}{*}{$\begin{array}{l}\text { Homework assignment } \\
\text { Television }\end{array}$} & \multicolumn{2}{|c|}{ Paper-and-pencil } & \multicolumn{2}{|c|}{ Memorization } & \multirow[b]{2}{*}{$N$} \\
\hline & $M$ & $S D$ & M & $S D$ & \\
\hline Soap opera & 40.43 & 12.06 & 38.58 & 13.05 & 40 \\
\hline Music videos & 35.03 & 11.48 & 35.80 & 14.04 & 40 \\
\hline Radio music & 36.05 & 9.75 & 36.10 & 13.92 & 40 \\
\hline Silence & 33.08 & 6.51 & 37.75 & 14.15 & 40 \\
\hline Total & 36.14 & 10.44 & 37.06 & 13.72 & 160 \\
\hline
\end{tabular}

trol condition, $t(39)=-3.50, p=.001$, and those of students in the radio condition, $t(39)=-3.28, p=.002$. No other $t$-test with Bonferroni correction reached statistical significance. The interaction between background condition and type of assignment was not statistically significant, $F(3,117)=.41, M S E=.02, p=.747, \eta^{2}=.002$.

The significant main effect found for background condition, combined with the absence of a significant interaction between background condition and type of assignment, confirmed Hypotheses 1 and 2. As anticipated in H1, participants who were exposed to a soap opera condition performed significantly worse than participants in the control condition, an effect that held not only for paper-andpencil assignments but also for memorization assignments. As anticipated in $\mathrm{H} 2$, students in the music videos condition performed about equally well as students in the control condition, a finding that applied both to paper-and-pencil and memorization assignments. $\mathrm{H} 3$, which predicted that distraction effects would be stronger for memorization than for paper-and-pencil assignments, was not confirmed, as there was no significant interaction between background condition and type of assignment. When memorization assignments were involved, students in the soap opera condition did not perform worse than students in the music videos condition, and there were no indications that students in the music videos condition performed worse than students in the radio condition. Thus, $\mathrm{H} 4$ and $\mathrm{H} 5$ were not confirmed.

\section{Homework Time}

Table 2 presents the mean number of minutes needed to complete the two types of assignments for each background condition. A 4 (background condition) X 2 (type of assignment) analysis of variance yielded no statistically significant main effect for type of assignment, $F(1,39)=.50, M S E=134.21, p=.485, \eta^{2}=.001$ : Students on average needed the same amount of time for the paper-and-pencil and memorization assignments. Also, no significant main effect was found for 
background condition, $F(3,117)=2.05, M S E=149.80, p=.111, \eta^{2}=.020$, indicating that the time used to complete the homework assignments was not dependent on the type of background medium. The interaction between background condition and type of assignment was not significant either, $F(3,117)=1.33, M S E=$ $113.39, p=.269, \eta^{2}=.010$, indicating that in each condition students on average needed the same amount of time to complete the memorization and paper-andpencil assignments. In conclusion, the results indicated that Hypotheses 1 through 5 were not confirmed, with the exception of $\mathrm{H} 2$, which predicted that music videos would not affect the amount of time spent on paper-and-pencil assignments.

\section{Discussion}

This study has unequivocally confirmed the main findings obtained in the study of Pool et al. (2000). In accordance with H1, and consistent with the results obtained in Pool et al.'s first experiment, watching a soap opera was found to impair concurrent performance on paper-and-pencil assignments. The proposition that soap operas interfere with students' homework performance gained additional strength from the observation that a background soap opera also impaired students' performance on memorization assignments. In agreement with Pool et al.'s (2000) findings, and as anticipated in the second hypothesis, foreign-language music videos in the background were found not to affect students' homework performance nor the amount of time it took to complete paper-and-pencil assignments. Moreover, there was no evidence to suggest that music videos negatively affected students' performance and time spent on memorization assignments.

Although the experiment was not designed to detect which psychological mechanism was responsible for the differential distraction effects found for soap operas and music videos, several theoretical explanations may be valid. First, capacity interference may account for the differences. Because soap operas offer coherent information that requires knowledge of preceding events, whereas music programs contain a series of unrelated music videos that are easily understandable and do not require any foreknowledge, soap operas probably make stronger demands on the students' limited attentional capacity. There is evidence that the demands made on attentional capacity are relatively small if the content of the message is easily understood (Lang, 1995).

Second, differential distraction effects found for the two program genres could result from differences in their potential for eliciting orienting responses that draw the attention from the task toward the screen (e.g., Anderson \& Lorch, 1983; Kahneman, 1973). When the student's visual attention is focused on the homework task, auditory stimuli can direct the student's attention from the homework to the screen, which leaves fewer resources to attend to the homework. Although music videos contain many visual characteristics that can elicit orienting responses, the auditory stimuli of soap operas probably invoke more orienting responses than the auditory stimuli of music videos because soap operas present new information, whereas music videos usually are very well known. In addition, students 
who combine doing homework with watching a soap opera have more reason to look at the screen from time to time because the pictorial information provided in this program type may be essential for understanding what is happening in the program. Rock music played in music videos, on the other hand, can be digested just as well without looking at the accompanying pictures.

Third, differential distraction effects could be due to structural interference because speech, which plays a dominant role in soap operas, interferes strongly with the phonological working memory also used to process the homework information (Armstrong \& Sopory, 1997; Salamé \& Baddeley, 1989). Processing the information in the background soap opera may leave insufficient capacity of the phonological working memory needed to process fully the information in the homework assignments.

Finally, differential distraction effects may have been caused by the different languages used in the two program types. It has been shown that background speech distracts more when using the native language rather than a foreign language (Martin et al., 1988). Therefore, the soap opera that used the native language of the participants (Dutch) may have caused stronger distraction effects than the music videos that employed a foreign language (English). The results found for music videos, therefore, cannot be generalized to the situation in which students combine doing homework with listening to music videos in their native language.

One addition to the Pool et al. (2000) study was that the impact of background music videos was compared with that of music radio. Results indicated that neither radio music nor music videos led to distraction effects. The only difference between the two music conditions was that music videos provided additional images. Apparently, the addition of television images to the music did not affect students' homework performance and time. Perhaps students in the music videos condition hardly looked at the television screen, and thus used television as a type of background radio, an assumption that can be checked when doing further research in which observational data are collected to assess students' viewing behavior.

The fact that we were unable to detect differences between the impact of background music videos and music radio does not mean that the medium does not matter. Our data suggest only that when foreign-language music programs are involved, the type of medium used makes no difference. For other program types that share a similar audio content, medium-specific distraction effects may occur, especially when the images shown on television provide interesting additions to the commentary or dialogue. In most cases, however, television programs are not directly comparable with radio programs because on television, part of the audio content of radio programs is conveyed through images rather than words. In these cases, it may be safely assumed that background television is more likely to interfere with homework performance than background radio because comprehension of the television program requires that at least part of the television images are watched.

A second new element in the present study was that the Pool et al. (2000) study was extended to include memorization tasks. Contrary to expectations, there were 
no indications that the distraction effects found varied with type of assignment. The distraction effects found for the soap opera held just as much for the paperand-pencil assignments as for the memorization tasks. In addition, our failure to detect significant distraction effects for music videos and music radio applied equally to both types of assignment. These findings cast doubt on the assumption that memorization tasks generally require more effort than paper-and-pencil assignments do. In the present study, we employed memorization tasks that requested students to memorize relatively simple factual information requiring little understanding or elaboration, which may have limited the demands made on students' attentional capacity. Therefore, it is questionable whether the memorization tasks in our study really occupied a greater amount of attentional capacity than the paper-and-pencil assignments did. The hypothesis that, relative to paperand-pencil assignments, memorization tasks are more likely to suffer from background media may hold true only if the material to be stored in memory is harder to understand and requires more elaboration.

Although students were matched on reading comprehension scores and gender, differences among conditions may have existed. For example, we did not measure students' IQs. However, the differences in IQs in the entire sample were small, as only students of the middle level of high school were included. In the Netherlands, students are placed in one of three high school levels, depending on their IQs. Another threat to the internal validity of the study could be the fact that individual students are more or less distracted by background media depending on some personality characteristic. Although students were randomly assigned to the background conditions to prevent potential unknown variables from threatening the internal validity, randomization does not exclude the possibility that differences between conditions remained.

A question related to the ecological validity of the present study is, to what extent the findings of a study conducted in a university building can be generalized to the home study situation. We selected a university setting for testing so we could control distraction and so that the observed differences between the experimental groups could be attributed confidently to the manipulated variables. The experimental setting of the present study more closely resembled home study in a student's bedroom than in a living room because in a living room other people may add to the distraction. However, this conclusion hardly limits the generalizability of our findings because about $80 \%$ of Dutch high school students usually do homework in their bedrooms (Beentjes et al., 1996).

The basic finding that soap operas cause distraction effects on homework performance when difficult tasks are involved, may be generalized to other television programs that are similar to soap operas. The fact that music videos did not cause distraction effects, whereas soap operas did, leads us to suspect that homework performance is hindered especially in combination with television programs that contain a lot of speech. Other program types that may hinder homework performance may therefore include situational comedies, high school series, and movies.

In conclusion, the findings of both the present experiment and the two experiments conducted by Pool et al. (2000) suggest that background music in a foreign language, irrespective of the medium used, is essentially harmless in practice. The 
presence of foreign-language background music left both the accuracy and the time used to complete paper-and-pencil and memorization assignments unaffected. However, because homework combined with watching a soap opera in the native language was found to impede performance, students should avoid combining homework with watching a soap opera, especially when difficult paper-and pencil and memorization assignments are involved.

\section{References}

Anderson, D. R., \& Lorch, E. P. (1983). Looking at television: Action or reaction? In J. Bryant \& D. R. Anderson (Eds.), Children's understanding of television: Research on attention and comprehension (pp. 1-33). New York: Academic Press.

Armstrong, G. B. (1997, May). Context and effects of background television on reading: A test of television effects on encoding, recall, and recognition. Paper presented at the annual conference of the International Communication Association, Montreal, Canada.

Armstrong, G. B., Boiarsky, G. A., \& Mares, M. L. (1991). Background television and reading performance. Communication Monographs, 58, 235-253.

Armstrong, G. B., \& Greenberg, B. S. (1990). Background television as an inhibitor of cognitive processing. Human Communication Research, 16, 355-386.

Armstrong, G. B., \& Sopory, P. (1997). Effects of background television on phonological and visuospatial working memory. Communication Research, 24, 459-506.

Basil, M. D. (1994). Multiple resource theory I: Application to television viewing. Communication Research, 21, 177-207.

Beentjes, J. W. J., Koolstra, C. M., \& van der Voort, T. H. A. (1996). Combining background media with doing homework: Incidence of background media use and perceived effects. Communication Education, 45, 59-72.

Boneau, C. A. (1960). The effects of violations of assumptions underlying the $t$ test. Psychological Bulletin, 57, 49-62.

Bourke, P. A., Duncan, J., \& Nimmo-Smith, I. (1996). A general factor involved in dual-task performance decrement. Quarterly Journal of Experimental Psychology, 49, 525-545.

Cook, R. D. (1977). Detection of influential observations in linear regression. Technometrics, 19, $15-18$.

Cook, R. D., \& Weisberg, S. (1982). Residuals and influence in regression. New York: Chapman \& Hall.

Cool, V. A., Yarbrough, D. B., Patton, J. E., Runde, R., \& Keith, T. Z. (1994). Experimental effects of radio and television distractors on children's performance on mathematics and reading assignments. Journal of Experimental Education, 62, 181-194.

Craik, F. I. M., \& Tulving, E. (1975). Depth of processing and the retention of words in episodic memory. Journal of Experimental Psychology: General, 104, 268-294.

Daoussis, L., \& McKelvie, S. J. (1986). Musical preferences and effects of music on a reading comprehension test for extraverts and introverts. Perceptual and Motor Skills, 62, 283-289.

Drew, D. G., \& Grimes, T. (1987). Audio-visual redundancy and TV news recall. Communication Research, 14, 452-461.

Feir-Walsh, B. J., \& Toothaker, L. E. (1974). An empirical comparison of the ANOVA F-test, normal scores test, and Kruskal-Wallis test under violations of assumptions. Educational and Psychological Measurement, 34, 789-799. 
Furnham, A., \& Bradley, A. (1997). Music while you work: The differential distraction of background music on the cognitive test performance of introverts and extraverts. Applied Cognitive Psychology, 11, 445-455.

Furnham, A., Gunter, B., \& Peterson, E. (1994). Television distraction and the performance of introverts and extraverts. Applied Cognitive Psychology, 8, 705-711.

Gilissen, J., \& Aarnoutse, C. (1996). Leesvaardighedentoets [Reading comprehension test]. Nijmegen, the Netherlands: Berkhout.

Graydon, J., \& Eysenck, M. W. (1989). Distraction and cognitive performance. European Journal of Cognitive Psychology, 1, 161-179.

Kahneman, D. (1973). Attention and effort. Englewood Cliffs, NJ: Prentice Hall.

Kirk, R. E. (1982). Experimental design: Procedures for the behavioral sciences (2nd ed.). Belmont, CA: Brooks/Cole.

Lang, A. (1995). Defining audio/video redundancy from a limited-capacity information processing perspective. Communication Research, 22, 86-115.

Lang, A. (2000). The limited capacity model of mediated message processing. Journal of Communication, 50, 46-70.

Levine, T. R., \& Hullett, C. R. (2000, June). Eta-square, partial eta-square, and misreporting of effect size in communication research. Paper presented at the annual conference of the International Communication Association, Acapulco, Mexico.

Martin, R. C., Wogalter, M. S., \& Forlano, J. G. (1988). Reading comprehension in the presence of unattended speech and music. Journal of Memory and Language, 27, 382-398.

Patton, J. E., Stinard, T. A., \& Routh, D. K. (1983). Where do children study? Journal of Educational Research, 76, 280-286.

Pool, M. M., van der Voort, T. H. A., Beentjes, J. W. J., \& Koolstra, C. M. (2000). Background television as an inhibitor of performance on easy and difficult homework assignments. Communication Research, 27, 293-326.

Salamé, P., \& Baddeley, A. D. (1989). Effects of background music on phonological short-term memory. Quarterly Journal of Experimental Psychology, 41, 107-122.

Stevens, J. (1992). Applied multivariate statistics for the social sciences (2nd ed.). Hillsdale, NJ: Erlbaum.

Tabachnick, B. G., \& Fidell, L. S. (1996). Using multivariate statistics (3rd ed.). New York: HarperCollins.

van Kan, N., \& Aarnoutse, C. (1993). Begrijpend leestest bestemd voor het eerste leerjaar van het MAVO, $H A V O$ en $V W O$ [Reading comprehension test intended for students in Grade 7]. Nijmegen, the Netherlands: Berkhout.

Wober, J. M. (1992). Text in a texture of television: Children's homework experience. Journal of Educational Television, 18, 23-34. 